\title{
UNIQUENESS OF QUASISTATIONARY DISTRIBUTIONS AND DISCRETE SPECTRA WHEN $\infty$ IS AN ENTRANCE BOUNDARY AND O IS SINGULAR
}

\author{
JORGE LITTIN C., ${ }^{*}$ Universidad de Chile
}

\begin{abstract}
We study quasistationary distributions on a drifted Brownian motion killed at 0 , when $+\infty$ is an entrance boundary and 0 is an exit boundary. We prove the existence of a unique quasistationary distribution and of the Yaglom limit.
\end{abstract}

Keywords: One-dimensional diffusion; entrance boundary; quasistationary distribution; exit boundary

2010 Mathematics Subject Classification: Primary $60 \mathrm{~J} 60$

Secondary 60G99

\section{Introduction}

We study quasistationary distributions (QSDs) for a drifted Brownian motion killed at 0 , when $+\infty$ is an entrance boundary according to Feller's classification. The most recent results for the existence and uniqueness of QSDs when 0 is a regular-type boundary are given in [7], and sufficient conditions are stated in [1] when 0 is an exit-type boundary.

This paper is related to [1]. We state the existence of a unique QSD when 0 is an exittype boundary and $+\infty$ is an entrance boundary, under the most general conditions. Also, the existence of the Yaglom limit is shown. In Section 2 the main hypothesis and some preliminary results are provided. In Section 3 the required elements of spectral theory are introduced. Finally, in Section 4 we summarize the main theorems on the existence of a unique QSD and the existence of the Yaglom limit.

The most technically difficult result is Theorem 3.2, which states the integrability of the eigenfunctions. The key to its proof is an increasing property of the eigenfunctions, which is established in (3.2).

\section{One-dimensional diffusion processes killed at the origin}

Let $X$ be a one-dimensional drifted Brownian motion in $(0, \infty)$, i.e.

$$
\mathrm{d} X_{t}=\mathrm{d} B_{t}-\alpha\left(X_{t}\right) \mathrm{d} t, \quad X_{0}=x>0,
$$

where $\alpha \in C^{1}(0, \infty)$ and $\left(B_{t}\right)_{t \geq 0}$ is a standard one-dimensional Brownian motion. We note that the drift $\alpha$ can explode at the origin. A pathwise solution of $X$ in (2.1) exists up to the explosion time $\tau$. We denote by $T_{y}$ the first time the process hits $y \in(0, \infty)$

Received 7 January 2011; revision received 10 January 2012.

* Postal address: Departamento Ingeniería Matemática and Centro Modelamiento Matemático, UMR 2071 CNRSUCHILE, Facultad Ciencias Físicas y Matemáticas, Universidad de Chile, Casilla 170, Correo 3, Santiago, Chile.

Email address: jlittin@dim.uchile.cl 
(see [5, Chapter VI, Section 3]) before the explosion, so $T_{y}=\inf \left\{0 \leq t<\tau: X_{t}=y\right\}$. We denote by

$$
T_{\infty}=\lim _{n \rightarrow \infty} T_{n} \quad \text { and } \quad T_{0}=\lim _{n \rightarrow \infty} T_{1 / n}
$$

Since $\alpha$ is regular in $(0, \infty), \tau=T_{0} \wedge T_{\infty}$.

\subsection{Main hypothesis}

Let $\gamma(x)=2 \int_{1}^{x} \alpha(z) \mathrm{d} z$ for $x \in(0, \infty)$. Associated to $\alpha$, we consider the following functions defined for $x \in(0, \infty)$ :

$$
\begin{aligned}
\Lambda_{1}(x) & =\int_{1}^{x} \mathrm{e}^{\gamma(z)} \mathrm{d} z, \\
\kappa(x) & =\int_{x}^{1} \mathrm{e}^{-\gamma(z)} \int_{x}^{z} \mathrm{e}^{\gamma(y)} \mathrm{d} y \mathrm{~d} z, \\
J(x) & =\int_{1}^{x} \mathrm{e}^{-\gamma(z)} \int_{1}^{z} \mathrm{e}^{\gamma(y)} \mathrm{d} y \mathrm{~d} z .
\end{aligned}
$$

Here $\Lambda_{1}(x)$ is the scale function and $\mu(\mathrm{d} x)=\mathrm{e}^{-\gamma(x)} \mathrm{d} x$ is the speed measure for $X$.

Observe that, under the condition $\alpha \in C^{1}(0, \infty), \gamma(y)$ is finite for all $y>0$, and both $\int_{a}^{b} \mathrm{e}^{\gamma(y)} \mathrm{d} y<\infty$ and $\int_{a}^{b} \mathrm{e}^{-\gamma(y)} \mathrm{d} y<\infty$ for all $0<a<b<\infty$.

Let us state the following conditions on $\alpha$.

(H1) Almost-sure absorption at 0: for all $x>0, \mathrm{P}_{x}\left(\tau=T_{0}<T_{\infty}\right)=1$.

(H2) $J(+\infty)<\infty$.

(H3) $\mu((0, \delta))=\infty$ for all $\delta>0$.

It is well known (see, for example, [5, Chapter VI, Theorem 3.2]) that (H1) holds if and only if $\Lambda_{1}(+\infty)=\infty$ and $\kappa\left(0^{+}\right)<\infty$. Also, note that (H1) can be written as $\mathrm{P}_{x}\left(\lim _{t \rightarrow \infty} X_{t \wedge \tau}=\right.$ $0)=1$.

As a direct consequence of hypotheses $(\mathrm{H} 1)$ and $(\mathrm{H} 2),+\infty$ is an entrance boundary according to Feller's classification (see [6, Chapter 15, Table 7.1] for details). Under hypotheses (H1) and (H3), 0 is an exit boundary according to Feller's classification (see [6, Chapter 15, Table 6.2] for details).

Hypothesis (H) is said to hold when $\alpha \in C^{1}(0, \infty)$ and (H1), (H2), and (H3) are satisfied. Note that, under $(\mathrm{H})$, the function

$$
\Lambda(x)=\int_{0}^{x} \mathrm{e}^{\gamma(z)} \mathrm{d} z \quad \text { for all } x \in(0, \infty)
$$

is finite. We also obtain some additional properties on the functions already defined, which we summarize in the next lemma.

Lemma 2.1. Assume that $(H)$ holds. Then the following relations are satisfied:

(i) $\Lambda_{1}(0+)>-\infty$,

(ii) $\mu((\delta, \infty))<\infty$ for all $\delta>0$,

(iii) $\int_{0}^{\infty} \mathrm{e}^{-\gamma(y)} \Lambda(y) \mathrm{d} y=\int_{0}^{\infty} \mathrm{e}^{\gamma(z)} \int_{z}^{\infty} \mathrm{e}^{-\gamma(y)} \mathrm{d} y \mathrm{~d} z<\infty$. 
Proof. (i) For all $0<\delta<1$, we have

$$
\left(\int_{0}^{\delta} \mathrm{e}^{\gamma(y)} \mathrm{d} y\right)\left(\int_{\delta}^{1} \mathrm{e}^{-\gamma(z)} \mathrm{d} z\right)<\int_{0}^{\delta} \mathrm{e}^{\gamma(y)}\left(\int_{y}^{1} \mathrm{e}^{-\gamma(z)} \mathrm{d} z\right) \mathrm{d} y<\kappa(0+)<\infty .
$$

Since $0<\int_{\delta}^{1} \mathrm{e}^{\gamma(z)} \mathrm{d} z<\infty$, the result is shown.

(ii) Since $\int_{\delta}^{M} \mathrm{e}^{-\gamma(y)} \mathrm{d} y<\infty$ for all $0<\delta<M<\infty$, it is enough to show that $\int_{M}^{\infty} \mathrm{e}^{-\gamma(y)} \mathrm{d} y<\infty$ for some $M>\delta$. From the condition $\Lambda_{1}(+\infty)=\infty$, there exists $M$ greater than 1 such that $\Lambda_{1}(x)>1$ for all $x>M$, so we have

$$
\int_{M}^{\infty} \mathrm{e}^{-\gamma(y)} \mathrm{d} y<\Lambda_{1}(M) \int_{M}^{\infty} \mathrm{e}^{-\gamma(y)} \mathrm{d} y<\int_{M}^{\infty} \mathrm{e}^{-\gamma(y)} \Lambda_{1}(y) \mathrm{d} y<J(+\infty)<\infty .
$$

(iii) From properties (i) and (ii), we have

$$
\begin{aligned}
\int_{0}^{\infty} \mathrm{e}^{-\gamma(y)} \Lambda(y) \mathrm{d} y & =\int_{0}^{1} \mathrm{e}^{-\gamma(z)} \Lambda(y) \mathrm{d} y+\int_{1}^{\infty} \mathrm{e}^{-\gamma(y)} \Lambda(y) \mathrm{d} y \\
& <\kappa(0+)+J(+\infty)+\left(\int_{1}^{\infty} \mathrm{e}^{-\gamma(y)} \mathrm{d} y\right)\left(\int_{0}^{1} \mathrm{e}^{\gamma(y)} \mathrm{d} y\right) \\
& <\infty .
\end{aligned}
$$

\subsection{Preliminary results}

Let

$$
\lambda^{*}:=\liminf _{t \rightarrow \infty}-\frac{\log \mathrm{P}_{x}\left(T_{0}>t\right)}{t}=\sup \left\{\lambda: \mathrm{E}_{x}\left(\mathrm{e}^{\lambda T_{0}}\right)<\infty\right\} .
$$

In fact, the right-hand equality follows from Fubini's theorem. Also, by irreducibility, both expressions on the right-hand side do not depend on $x$ (see claim 1 in the proof of Theorem 2.1 below), so $\lambda^{*}$ is well defined.

The next lemma gives some additional information about $\lambda^{*}$ when $+\infty$ is an entrance boundary.

Lemma 2.2. Assume that (H) holds. Then

$$
\lambda^{*}>\frac{1}{2 \int_{0}^{\infty} \mathrm{e}^{\gamma(z)} \int_{z}^{\infty} \mathrm{e}^{-\gamma(y)} \mathrm{d} y \mathrm{~d} z}>0
$$

Proof. The proof is analogous to that of Proposition (7.6) of [1]. We have $J_{\delta}(x)=$ $\int_{0}^{x} \mathrm{e}^{\gamma(y)} \mathrm{d} y \int_{y}^{\infty} \mathrm{e}^{-\gamma(z)} \mathrm{d} z+\delta$, with $\delta>0$. From $(\mathrm{H} 2), J_{\delta}(\infty)<\infty$, and from a straightforward computation we get $\mathcal{L} J_{\delta}=\frac{1}{2} J_{\delta}^{\prime \prime}-J_{\delta}^{\prime}=-\frac{1}{2}$ and $\int_{\varepsilon}^{1 / \varepsilon}\left|J_{\delta}^{\prime}(s)\right|^{2} \mathrm{~d} s<\infty$ for all $\varepsilon>0$.

By Itô's formula,

$$
\mathrm{E}_{x}\left(\mathrm{e}^{a T_{\varepsilon} \wedge T_{1 / \varepsilon} \wedge t} J_{\delta}\left(X_{T_{\varepsilon} \wedge T_{1 / \varepsilon} \wedge t}\right)\right)=J_{\delta}(x)+\mathrm{E}_{x}\left(\int_{0}^{T_{\varepsilon} \wedge T_{1 / \varepsilon} \wedge t} \mathrm{e}^{a s}\left(a J_{\delta}\left(X_{S}\right)+\mathcal{L} J_{\delta}\left(X_{s}\right)\right) \mathrm{d} s\right) .
$$

For $0<a \leq 1 / 2 J_{\delta}(+\infty)$, it is clear that $J_{\delta}\left(X_{s}\right)+\mathcal{L} J_{\delta}\left(X_{s}\right) \leq 0$, so

$$
\delta \mathrm{E}_{x}\left(\mathrm{e}^{a T_{\varepsilon} \wedge T_{1 / \varepsilon} \wedge t}\right) \leq \mathrm{E}_{x}\left(\mathrm{e}^{a T_{\varepsilon} \wedge T_{1 / \varepsilon} \wedge t} J_{\delta}\left(X_{T_{\varepsilon} \wedge T_{1 / \varepsilon} \wedge t}\right)\right) \leq J_{\delta}(x) .
$$

Let $t \rightarrow \infty$ and $\varepsilon \rightarrow 0$. From the monotone convergence theorem we obtain

$$
\mathrm{E}_{x}\left(\mathrm{e}^{a T_{0}}\right) \leq \frac{J_{\delta}(x)}{\delta}<\infty \quad \text { for all } a \in\left(0, \frac{1}{2 J_{\delta}(\infty)}\right]
$$

Finally, since the result is true for all $\delta>0$, we conclude the proof by taking $\delta \rightarrow 0^{+}$. 
We recall that a probability measure $v$ is a QSD if $\mathrm{E}_{v}\left(X_{t} \in A \mid T_{0}>t\right)=v(A)$ for all Borel subsets $A \subseteq(0, \infty)$. It is known (see [2] and [3]) that if $v$ is a QSD then $\mathrm{P}_{v}\left(T_{0}>t\right)=\mathrm{e}^{-\lambda(v) t}$ for some $0<\lambda(v) \leq \lambda^{*}$, where $\lambda(v)$ is the survival rate of $T_{0}$ starting from $v$. Then, each QSD is necessarily associated to a $\lambda \in\left(0, \lambda^{*}\right]$. Theorem 2.1 below shows that, when $\infty$ is an entrance boundary, any QSD $v$ satisfies $\lambda(v)=\lambda^{*}$, but before presenting this result we recall the next definition.

Definition 2.1. $X$ comes down from $\infty$ if, for some $y>0, \lim _{x \rightarrow \infty} \mathrm{P}_{x}\left(T_{y} \leq t\right)>0$.

Theorem 2.1. Let hypothesis $(H)$ hold. Then no $Q S D$ is associated to some $\lambda \in\left(0, \lambda^{*}\right)$.

Proof. We verify this result by proving the following four claims.

Claim 2.1. If $\mathrm{E}_{x_{0}}\left(\mathrm{e}^{\lambda T_{0}}\right)=\infty$ for some $x_{0}>0$ then $\mathrm{E}_{x}\left(\mathrm{e}^{\lambda T_{0}}\right)=\infty$ for all $x>0$.

Proof. For $x>x_{0}$, the claim follows straightforwardly since $\mathrm{E}_{x}\left(\mathrm{e}^{\lambda T_{0}}\right)>\mathrm{E}_{x_{0}}\left(\mathrm{e}^{\lambda T_{0}}\right)$. For $0<x<x_{0}$, we have

$$
\mathrm{E}_{x}\left(\mathrm{e}^{\lambda T_{0}}\right)>\mathrm{E}_{x}\left(\mathrm{e}^{\lambda T_{0}} \mathbf{1}_{\left\{T_{0}>T_{x_{0}}\right\}}\right)=\mathrm{E}_{x}\left(\mathrm{e}^{\lambda T_{x_{0}}} \mathbf{1}_{\left\{T_{0}>T_{x_{0}}\right\}}\right) \mathrm{E}_{x_{0}}\left(\mathrm{e}^{\lambda T_{0}}\right) \geq \mathrm{P}_{x}\left(T_{x_{0}}<T_{0}\right) \mathrm{E}_{x_{0}}\left(\mathrm{e}^{\lambda T_{0}}\right) .
$$

Since $0<\mathrm{P}_{x}\left(T_{x_{0}}<T_{0}\right)=\Lambda(x) / \Lambda\left(x_{0}\right)<1$, the claim follows.

Claim 2.2. Let $x_{0}>0$ and $\lambda>0$ such that $\mathrm{E}_{x_{0}}\left(\mathrm{e}^{\lambda T_{0}}\right)<\infty$. Then $X$ comes down from $\infty$ if and only if $\sup _{x>0} \mathrm{E}_{x}\left(\mathrm{e}^{\lambda T}\right)<\infty$.

Proof. Let us prove the 'if' part. We know that $\mathrm{E}_{x}\left(\mathrm{e}^{\lambda T}\right)<\infty$ for all $x>0$. From [1, Proposition 7.6] we know that, for all $\lambda>0$, there exists $y_{\lambda}$ such that $\sup _{x>y_{\lambda}} \mathrm{E}_{x}\left(\mathrm{e}^{\lambda T_{y_{\lambda}}}\right)<\infty$. By monotonicity and the strong Markov property, $\sup _{x>0} \mathrm{E}_{x}\left(\mathrm{e}^{\lambda T_{0}}\right)<\infty$. have

Let us now prove the 'only if' part. Let $y>x>0$. By using the Markov inequality we

$$
\mathrm{P}_{x}\left(T_{y}>t\right) \leq \mathrm{e}^{-\lambda t} \mathrm{E}_{x}\left(\mathrm{e}^{\lambda T_{y}}\right) \leq \mathrm{e}^{-\lambda t} \sup _{x>0} \mathrm{E}_{x}\left(\mathrm{e}^{\lambda T_{y}}\right)<1
$$

for large enough $t$. The latter implies that $X$ comes down from $\infty$ (see Definition 2.1). This completes the proof of the claim.

Claim 2.3. Assume that $(H)$ holds. If $\lambda>0$ satisfies $\sup _{x>0} \mathrm{E}_{x}\left(\mathrm{e}^{\lambda T}\right)<\infty$ then there does not exist a QSD associated to $\lambda$.

Proof. Assume that $\pi_{\lambda}$ is a QSD associated to $\lambda$. Then $\mathrm{P}_{\pi_{\lambda}}\left(T_{0}>t\right)=\mathrm{e}^{-\lambda t}$. Now, for all $t>0$, it is satisfied:

$$
\begin{aligned}
\infty & >\sup _{x>0} \mathrm{E}_{x}\left(\mathrm{e}^{\lambda T_{0}}\right) \\
& \geq \mathrm{E}_{\pi_{\lambda}}\left(\mathrm{e}^{\lambda T_{0}}\right) \\
& =\int_{0}^{\infty} \mathrm{E}_{x}\left(\mathrm{e}^{\lambda T_{0}}\right) \pi_{\lambda}(\mathrm{d} x) \\
& \geq \lambda \int_{0}^{\infty}\left(\int_{0}^{t} \mathrm{e}^{\lambda s} \mathrm{P}_{x}\left(T_{0}>s\right) \mathrm{d} s\right) \pi_{\lambda}(\mathrm{d} x) .
\end{aligned}
$$

By Fubini's theorem, the term on the right-hand side becomes

$$
\lambda \int_{0}^{t}\left(\int_{0}^{\infty} \pi_{\lambda}(\mathrm{d} x) \mathrm{P}_{x}\left(T_{0}>s\right)\right) \mathrm{e}^{\lambda s} \mathrm{~d} s=\lambda \int_{0}^{t} \mathrm{e}^{-\lambda s} \mathrm{e}^{\lambda s} \mathrm{~d} s=\lambda t .
$$

The inequality holds for $t>0$, which is a contradiction, and so the claim is proven. 
Claim 2.4. If $X$ comes down from $\infty$ then, for all $\lambda \in\left(0, \lambda^{*}\right)$, we have $\sup _{x>0} \mathrm{E}\left(\mathrm{e}^{\lambda T_{0}}\right)<\infty$.

Proof. From claims 2 and 3 , it is sufficient to show that, for $\lambda \in\left(0, \lambda^{*}\right), \mathrm{E}_{x}\left(\mathrm{e}^{\lambda T_{0}}\right)<\infty$ holds for some (for all) $x>0$. From (the lim inf) definition in (2.2), for all $\varepsilon>0$, there exists $t_{0}$ such that, for all $t>t_{0}$, it holds that $\lambda^{*}>-\log \mathrm{P}_{x}(T>t) / t>\lambda^{*}-\varepsilon$, or, equivalently, $\mathrm{P}_{x}(T>t)<\mathrm{e}^{-\left(\lambda^{*}-\varepsilon\right) t}$ for all $t>t_{0}$. By choosing $0<\varepsilon<\lambda^{*}-\lambda$ we obtain

$$
\begin{aligned}
\mathrm{E}_{x}\left(\mathrm{e}^{\lambda T_{0}}\right) & =1+\lambda \int_{0}^{\infty} \mathrm{e}^{\lambda s} \mathrm{P}_{x}\left(T_{0}>s\right) \mathrm{d} s \\
& =1+\lambda\left(\int_{0}^{t_{0}} \mathrm{e}^{\lambda s} \mathrm{P}_{x}\left(T_{0}>s\right) \mathrm{d} s+\int_{t_{0}}^{\infty} \mathrm{e}^{\lambda s} \mathrm{P}_{x}\left(T_{0}>s\right) \mathrm{d} s\right) \\
& <1+\lambda t_{0} \mathrm{e}^{\lambda t_{0}}+\int_{t_{0}}^{\infty} \mathrm{e}^{\left(\lambda-\lambda^{*}+\varepsilon\right) s} \mathrm{~d} s \\
& <\infty,
\end{aligned}
$$

proving the claim.

This completes the proof of Theorem 2.1.

\section{3. $L^{2}$ and spectral theory of the diffusion process}

\subsection{Spectral theory and the Sturm-Liouville problem}

As in [1], we will give an $L^{2}$ version of the semigroup $P_{t}$ and its associated generator $\mathcal{L}$. The analysis is based on the theory of the Sturm-Liouville problem, which has recently been studied in detail in [8].

Let $\mu$ be the speed measure of the process. For $f, g \in L^{2}(\mu)$, define

$$
(f, g)_{\mu}=\int_{0}^{\infty} f(s) g(s) \mu(\mathrm{d} s) .
$$

Let $\mathrm{AC}_{\text {loc }}(0, \infty)$ be the space of locally absolutely continuous functions on $(0, \infty)$, let $D_{\max }=$ $\left\{f, \mathrm{e}^{\gamma}\left(\mathrm{e}^{-\gamma} f^{\prime}\right)^{\prime} \in \mathrm{AC}_{\text {loc }}(0, \infty) \cap L^{2}(\mu)\right\}$, and let $D_{0}=\left\{f \in D_{\max }\right.$ : has compact support $\}$. Consider the following operators on $L^{2}(\mu): \mathcal{L}_{\max } f=-\frac{1}{2} \mathrm{e}^{\gamma}\left(\mathrm{e}^{-\gamma} f^{\prime}\right)^{\prime}$ for $f \in D_{\max }$ and $\mathcal{L}_{0} f=-\frac{1}{2} \mathrm{e}^{\gamma}\left(\mathrm{e}^{-\gamma} f^{\prime}\right)^{\prime}$ for $f \in D_{0}$. Note that these expressions are defined $\mu$-almost everywhere in both cases. For $f, g$ in $D_{0},\left(\mathscr{L}_{0} f, g\right)_{\mu}=\left(f^{\prime}, g^{\prime}\right)_{\mu}$ is a symmetric form on $D_{0}$.

Following Lemma 10.3.1 of [8], let $D_{\min }$ and $D_{\max }$ be dense in $L^{2}(\mu)$, let $\mathcal{L}_{0}$ be closable so that its closure, denoted by $\mathscr{L}_{\text {min }^{-}}$, is closed, symmetric, and densely defined (on $D_{0}$ ), and let $\mathcal{L}_{\text {min }}$ and $\mathcal{L}_{\text {max }}$ satisfy $\mathcal{L}_{\text {min }}^{*}=\mathcal{L}_{\text {max }}$ and $\mathcal{L}_{\text {max }}^{*}=\mathcal{L}_{\text {min }}$. Then, for any self-adjoint extension $\mathcal{L}$ of $\mathcal{L}_{\text {min }}$, we have $\mathcal{L}_{\text {min }} \subseteq \mathcal{L}=\mathcal{L}^{*} \subseteq \mathcal{L}_{\max }$.

We will see that in our case, $\mathcal{L}_{\min }$ is itself a self-adjoint operator with no proper self-adjoint extensions on $L^{2}(\mu)$, i.e. if $\mathcal{L}$ is a self-adjoint extension of $\mathcal{L}_{\min }$, it satisfies $\mathcal{L}_{\min }=\mathcal{L}=\mathcal{L}^{*}=$ $\mathcal{L}_{\text {max }}$. For this purpose, it is necessary to give an appropriate classification of the endpoints 0 and $\infty$. We introduce the following notions for the endpoint 0 .

Definition 3.1. (i) 0 is a regular endpoint if $\int_{0}^{d} \mathrm{e}^{-\gamma(s)} \mathrm{d} s<\infty$ holds for some (and, therefore, for all) $d>0$. If an endpoint is not regular, it is called singular.

(ii) 0 is a limit circle (LC) endpoint if all the solutions of the equation $-\frac{1}{2} \mathrm{e}^{\gamma}\left(\mathrm{e}^{-\gamma} f^{\prime}\right)^{\prime}=\lambda f$, $\lambda \in \mathbb{C}$, are in $L^{2}(\mu,(0, d))$ for some $0<d<\infty$. If 0 is not an LC, it is called a limit point (LP). 
(iii) 0 is an oscillatory $(\mathrm{O})$ endpoint if there exists a nontrivial real-valued solution to $-\frac{1}{2} \mathrm{e}^{\gamma}\left(\mathrm{e}^{-\gamma} f^{\prime}\right)^{\prime}=\lambda f, \lambda \in \mathbb{R}$, with an infinite number of 0 s in any neighborhood of the origin. Otherwise, we say that 0 is a nonoscillatory (NO) endpoint.

Similar definitions hold for $+\infty$. We recall that the LC/LP classifications are independent of $\lambda$, but the $\mathrm{O} / \mathrm{NO}$ classifications depend on $\lambda$ in general. It is clear that, under hypothesis $(\mathrm{H})$, both endpoints are singular; in fact,

$$
\int_{0}^{d} \mathrm{e}^{-\gamma(s)} \mathrm{d} s=\infty \text { and } \int_{d}^{\infty} \mathrm{e}^{\gamma(s)} \mathrm{d} s=\infty \quad \text { for all } 0<d<\infty .
$$

In the next result we show that 0 and $\infty$ are also LP endpoints.

Lemma 3.1. Assume that $(H)$ holds. Then 0 and $+\infty$ are of LP type.

Proof. Since the classification does not depend on the value of $\lambda$, we will use $\lambda=0$. In this case, the solutions are linear combinations of $f_{1}(x)=1$ and $f_{2}(x)=\Lambda(x)$. The case 0 is trivial since $\int_{0}^{d}\left|f_{1}(s)\right|^{2} \mathrm{e}^{-\gamma(s)} \mathrm{d} s=\int_{0}^{d} \mathrm{e}^{-\gamma(s)} \mathrm{d} s=\infty$ for all $d>0$. For the $+\infty$ case, note that $\int_{d}^{\infty}\left(\mathrm{e}^{\gamma(s)} / \Lambda(s)^{2}\right) \mathrm{d} s=1 / \Lambda(d)<\infty$ and $0<\mathrm{e}^{-\gamma(d)} \Lambda^{2}(d)<\infty$ for all $0<d<\infty$. Then, for $M>0$, we have

$$
\begin{aligned}
M & =\int_{d}^{M+d}\left(\mathbf{1}_{\left\{\mathrm{e}^{-\gamma(s)} \Lambda^{2}(s)>1\right\}}+\mathbf{1}_{\left\{\mathrm{e}^{-\gamma(s)} \Lambda^{2}(s) \leq 1\right\}}\right) \mathrm{d} s \\
& \leq \int_{d}^{M+d} \mathrm{e}^{-\gamma(s)} \Lambda^{2}(s) \mathrm{d} s+\int_{d}^{M+d} \frac{\mathrm{e}^{\gamma(s)}}{\Lambda(s)^{2}} \mathrm{~d} s \\
& \leq \int_{d}^{\infty} \mathrm{e}^{-\gamma(s)} \Lambda^{2}(s) \mathrm{d} s+\frac{1}{\Lambda(d)} .
\end{aligned}
$$

Since the inequality holds for all $M>0$, letting $M \rightarrow \infty$ we conclude that the integral on the right-hand side diverges.

Theorem 10.4.1 of [8] states that if (and only if) 0 and $\infty$ are LP endpoints then $\mathcal{L}_{\min }$ is itself a self-adjoint operator and has no proper self-adjoint extensions on $L^{2}(\mu)$ (see above). We conclude that $\mathcal{L}_{0}$ is a symmetric, closable, densely defined operator on $L^{2}(\mu)$, whose smallest closure $\mathcal{L}_{\text {min }}$ (denoted by $\mathcal{L}$ in the sequel) is a self-adjoint operator with no proper extensions, and it is also Markovian. Hence, $\mathcal{L}$ is a regular Dirichlet form and possesses the local property (see, for example, [1] and [4, Theorem 2.1.2]). We recall that the approach is the same as that in [1] if $D_{0}$ is replaced by $C_{0}^{\infty}(0, \infty)$ (which is included in $D_{0}$ ). Then, Theorem 6.2.2 of [4] applied as in [1] gives the existence of a nonpositive self-adjoint operator $\mathcal{L}$ on $L^{2}(\mu)$ with domain $D(\mathcal{L}) \supseteq C_{0}^{\infty}((0, \infty))$ such that, for $f, g \in C_{0}^{((0, \infty))}, \mathcal{E}(f, g)=-2(f, \mathcal{L} g)_{\mu}$ (see $[4$, Theorem 1.3.1]). We point out that, for $g \in C_{0}^{\infty}((0, \infty)), \mathcal{L} g=\frac{1}{2} g^{\prime \prime}-\alpha g^{\prime}$. Moreover, $\mathcal{L}$ is the generator of a strongly continuous symmetric semigroup of contractions on $L^{2}(\mu)$ denoted by $\left(P_{t}: t \geq 0\right)$. This semigroup is sub-Markovian, that is, $0 \leq P_{t} \leq 1, \mu$-almost everywhere if $0 \leq f \leq 1$ (see [4, Theorem 1.4.1]).

Following [1], it can be shown that the semigroup $P_{t}$ and the semigroup induced by the strong Markov process $\left(X_{t \wedge \tau}\right)$ coincides on the set of smooth and compactly supported functions. Also, from [1] we know that, when absorption is sure, that is, (H1) holds, the semigroup coincides with the semigroup of $X$ killed at 0 , that is, $P_{t} f(x)=\mathrm{E}_{x}\left(f\left(X_{t}\right) \mathbf{1}_{\left\{T_{0}>t\right\}}\right)$.

Now we will show the discreteness of the spectrum and the main theorem of this section. 
Proposition 3.1. Assume that $(H)$ holds. Then $-\mathcal{L}$ has a purely discrete spectrum. The eigenvalues

$$
-\infty<\lambda_{0}<\lambda_{1}<\cdots<\lambda_{2}<\cdots
$$

are simple, $\lim _{n \rightarrow \infty} \lambda_{n}=+\infty$, and the eigenfunction $\psi_{n}$ associated to $\lambda_{n}$ has exactly $n$ roots belonging to $(0, \infty)$ and a orthonormal basis of $L^{2}(\mu)$. In particular, we can take $\psi_{\lambda_{0}}$ strictly positive.

Proof. Recall that we are analyzing the nontrivial solutions of the equation

$$
\left(\mathrm{e}^{-\gamma} \psi_{\lambda}^{\prime}\right)^{\prime}=-2 \lambda \mathrm{e}^{-\gamma} \psi_{\lambda} .
$$

Let $\sigma_{\mathrm{e}}=\sigma_{\mathrm{e}}(\mathcal{L})$ denote the essential spectrum and $\sigma_{\mathrm{d}}=\sigma_{\mathrm{d}}((L))$ denote the discrete part of the spectrum. From part 8 of Theorem 10.4.1 of [8], we know that if (at least) one endpoint is LP, then either $\sigma_{\mathrm{e}}$ or $\sigma_{\mathrm{d}}$ is nonempty. Let $\sigma_{0}=\inf \sigma_{\mathrm{e}}$. The result will be shown once we prove that $\sigma_{0}=+\infty$. Since $\sigma_{0}$ is such that the all the nontrivial solutions of $\mathcal{L} \psi_{\lambda}=-\lambda \psi_{\lambda}$ are NO for $\lambda<\sigma_{0}$ and $\mathrm{O}$ for $\lambda<\sigma_{0}$, we need to prove that the solutions are NO for each $\lambda>0$.

Using the same argument as in Theorem 3.16 of [7], we find that between a local minimum $\underline{x}_{i}$ and a local maximum $\bar{x}_{i} \in(0, \infty)$, there exists exactly one solution to the equation $\psi_{\lambda}(x)=0$, and also $\psi_{\lambda}\left(\underline{x}_{i}\right)<0$ and $\psi_{\lambda}\left(\bar{x}_{i}\right)>0$. Moreover, for each pair of consecutive $\underline{x}_{i}$ and $\bar{x}_{i}$ local extrema, we have

$$
\frac{1}{2 \lambda}<\int_{\underline{x}_{i}}^{\bar{x}_{i}} \Lambda(s) \mathrm{e}^{-\gamma(s)} \mathrm{d} s .
$$

Then the relation

$$
\int_{0}^{\infty} \Lambda(s) \mathrm{e}^{-\gamma(s)} \mathrm{d} s>\int_{\varepsilon}^{\infty} \Lambda(s) \mathrm{e}^{-\gamma(s)} \mathrm{d} s>\sum_{\left(\underline{x}_{i}, \bar{x}_{i}\right)} \int_{\underline{x}_{i}}^{\bar{x}_{i}} \Lambda(s) \mathrm{e}^{-\gamma(s)} \mathrm{d} s \geq \frac{1}{2 \lambda} \#\left(\underline{x}_{i}, \bar{x}_{i}\right)
$$

is satisfied for all $\varepsilon>0$, where $\#\left(\underline{x}_{i}, \bar{x}_{i}\right)$ denotes the number of solutions to $\psi_{\lambda}(x)=0$ in $(\varepsilon, \infty)$. So, the number of roots in $[\varepsilon, \infty)$ is bounded uniformly by $2 \lambda \int_{0}^{\infty} \Lambda(s) \mathrm{e}^{-\gamma(s)} \mathrm{d} s+1$. The result follows by letting $\varepsilon \rightarrow 0^{+}$.

A direct consequence of the previous proof is the inequality $\lambda_{n} \geq n /\left(2 \int_{0}^{\infty} \Lambda(s) \mathrm{e}^{-\gamma(s)} \mathrm{d} s\right)$. Indeed, it is implied by the fact that $\psi_{\lambda_{n}}$ has exactly $n$ roots. Also, we can erase the term +1 if we already know that $\psi_{\lambda}$ has a finite number of 0 s.

Theorem 3.1. It holds that

$$
\begin{aligned}
\left(P_{t} f, g\right)_{\mu} & =\sum_{i \geq 0} \mathrm{e}^{-\lambda_{i} t}\left(\psi_{\lambda_{i}}, f\right)_{\mu}\left(\psi_{\lambda_{i}}, g\right)_{\mu} \quad \text { for all } f, g \in L^{2}(\mu), \\
P_{t} f & =\sum_{i \geq 0} \mathrm{e}^{-\lambda_{i} t}\left(\psi_{\lambda_{i}}, f\right)_{\mu} \psi_{\lambda_{i}}(x) \quad \text { for all } f \in L^{2}(\mu), \\
\lim _{t \rightarrow \infty} \mathrm{e}^{\lambda_{0} t}\left(g, P_{t} f\right)_{\mu} & =\left(\psi_{\lambda_{0}}, f\right)_{\mu}\left(\psi_{\lambda_{0}}, g\right)_{\mu} \text { for all } f, g \in L^{2}(\mu), f \geq 0, g \geq 0 .
\end{aligned}
$$

Proof. It is straightforward from the $L^{2}$ version of the process.

Theorem 3.1 is similar to Theorem 3.2 of [1], with the main difference being that here we do not impose extra conditions on $\alpha$ to get a discrete spectrum. In fact, we only assume regularity on $\alpha$ to guarantee the existence of a diffusion process, a hypothesis that is often assumed to avoid technical problems. 
The next step is to prove the existence (and, as we will see below, also uniqueness) of QSDs. The function $\mathrm{e}^{-\gamma} \psi_{\lambda_{0}}$ is a natural candidate for being a QSD; in fact, for all $f \in L^{2}(\mu)$, we have

$$
\left(\psi_{\lambda_{0}}, P_{t} f\right)_{\mu}=\left(\psi_{\lambda_{0}}, f\right)_{\mu} .
$$

Then, $\mathrm{e}^{-\gamma} \psi_{\lambda_{0}}$ satisfies the necessary condition on a restricted set of functions. We need to prove the following two facts: (i) $\psi_{\lambda_{0}} \in L^{1}(\mu)$ and (ii) $\lambda_{0}>0$. The latter assertion follows from (3.1). In fact, since $\psi_{\lambda_{0}}$ is a positive element in $L^{2}(\mu)$, we can take $f=g=\psi_{\lambda_{0}}$ in (3.1) to obtain

$$
0=\lim _{t \rightarrow \infty}\left(P_{t} \psi_{\lambda_{0}}, \psi_{\lambda_{0}}\right)_{\mu}=\lim _{t \rightarrow \infty} \mathrm{e}^{-\lambda_{0} t}\left|\psi_{\lambda_{0}}\right|_{L^{2}(\mu)}^{2} .
$$

This implies that $\lambda_{0}>0$.

It remains to prove the integrability of $\psi_{\lambda_{0}}$. In Theorem 3.2 we will prove that all the eigenfunctions are in $L_{1}(\mu)$. First, let us collect some elementary properties of the eigenfunctions.

- $+\infty$ is an LP, and from [8, Lemma 10.4.1] we know that, for all $g \in D_{\max }$, $\lim _{x \rightarrow \infty} \mathrm{e}^{-\gamma}\left[\psi_{\lambda_{k}}^{\prime} g-g^{\prime} \psi_{\lambda_{k}}\right](x)=0$. If we choose $g \in D_{\max }$ such that $g(x)=1$ for large $x$ (the existence of such a $g$ is guaranteed by $\int_{1}^{\infty} 1^{2} \mathrm{e}^{-\gamma(s)}<\infty$ ), we obtain $\lim _{x \rightarrow \infty} \mathrm{e}^{-\gamma} \psi_{\lambda_{k}}^{\prime}(x)=0$.

- Since $\psi_{\lambda_{0}}>0$, and it satisfies $\left(\mathrm{e}^{-\gamma(x)} \psi_{\lambda_{0}}^{\prime}(x)\right)^{\prime}=-2 \lambda_{0} \mathrm{e}^{-\gamma(x)} \psi_{\lambda_{0}}(x), x>0$, we obtain $\mathrm{e}^{-\gamma(x)} \psi_{\lambda_{0}}^{\prime}(x)>\lim _{x \rightarrow \infty} \mathrm{e}^{-\gamma} \psi_{\lambda_{0}}^{\prime}(x)=0$. In particular, $\psi_{\lambda_{0}}$ is an increasing function.

- The last assertion implies the existence of the limit $\psi_{\lambda_{0}}(0+)=\lim _{x \rightarrow 0} \psi_{\lambda_{0}}(x)$ and also that its value is 0 . In fact, if the limit is greater than 0 we obtain $\int_{0}^{1} \psi_{\lambda_{0}}^{2}(s) \mathrm{e}^{-\gamma(s)} \mathrm{d} s>$ $\psi_{\lambda_{0}}^{2}(0+) \int_{0}^{1} \mathrm{e}^{-\gamma(s)} \mathrm{d} s=\infty$, which is a contradiction.

For $\psi_{\lambda_{i}}, i \geq 1$, we can state similar results; in fact, without loss of generality, we can suppose that $\psi_{\lambda_{i}}>0$ in $\left(0, x_{1, i}\right)$, where $x_{1, i}$ denotes the smallest positive solution of $\psi_{\lambda_{i}}=0$, in which case $\psi_{\lambda_{i}}^{\prime}\left(x_{1, i}\right)<0$ (or, equivalently, $\left.\mathrm{e}^{-\gamma\left(x_{1, i}\right)} \psi_{\lambda_{i}}^{\prime}\left(x_{1, i}\right)<0\right)$ and $\mathrm{e}^{-\gamma} \psi_{\lambda_{i}}^{\prime}$ decreases in $\left(0, x_{1, i}\right)$. Moreover, there exists $0<x_{0, i}^{*}<x_{1, i}$ such that $\mathrm{e}^{-\gamma} \psi_{\lambda_{i}}^{\prime}\left(x_{0, i}^{*}\right)=0$ (because otherwise we obtain $\psi_{\lambda_{i}}(0+)>0$, which is a contradiction). Then, for $x \in\left(0, x_{0, i}^{*}\right), \psi_{\lambda_{i}}^{\prime}(x)>\psi_{\lambda_{i}}^{\prime}\left(x_{0, i}^{*}\right)=0$, so we conclude that $\psi_{\lambda_{i}}$ is positive and increasing for some neighborhood of 0 .

\subsection{Integrability of the eigenfunctions}

First, let us note that, for all $\delta>x$ and $k \geq 0$,

$$
\begin{aligned}
\int_{x}^{\infty}\left|\psi_{\lambda_{i}}(s)\right| \mathrm{e}^{-\gamma(s)} \mathrm{d} s & \leq \sqrt{\int_{x}^{\infty} \mathrm{e}^{-\gamma(y)} \mathrm{d} y} \sqrt{\int_{x}^{\infty} \psi_{\lambda_{i}}(y)^{2} \mathrm{e}^{-\gamma(y)} \mathrm{d} y} \\
& <\sqrt{\int_{x}^{\infty} \mathrm{e}^{-\gamma(y)} \mathrm{d} y} \\
& <\infty
\end{aligned}
$$

Then, $\int_{0}^{\infty}\left|\psi \lambda_{i}(s)\right| \mathrm{e}^{-\gamma(s)} \mathrm{d} s$ converges if and only if $\int_{0}^{x}\left|\psi_{\lambda_{i}}(s)\right| \mathrm{e}^{-\gamma(s)} \mathrm{d} s$ for some (and, therefore, for all) $x>0$. The next theorem shows that, in fact, all the eigenfunctions are absolutely integrable with respect to the measure $\mu$.

Theorem 3.2. Let hypothesis $(H)$ hold. Then, for all $k \geq 0, \psi_{\lambda_{k}} \in L^{1}(\mu)$. 
Proof. Let us recall the equation

$$
\left(\mathrm{e}^{-\gamma(x)} \psi_{\lambda_{i}}^{\prime}(x)\right)^{\prime}=-2 \lambda_{i} \mathrm{e}^{-\gamma(x)} \psi_{\lambda_{i}}(x)
$$

Let $x_{0,0}=\hat{x}_{0,0}^{*}$ and $x_{0, i}=\min \left(x_{0, i}^{*}, \hat{x}_{0, i}^{*}\right)$, where $\hat{x}_{0, i}^{*}$ is the solution to the equation $2 \lambda_{i} \int_{0}^{x} \mathrm{e}^{-\gamma(s)} \Lambda(s) \mathrm{d} s=1$, and $x_{0, i}^{*}$ denotes the smallest solution to $\mathrm{e}^{-\gamma} \psi_{\lambda_{i}}^{\prime}(x)=0$.

Take $g \in D_{\max }$ such that $g(x)=-\Lambda(x)$ for $x \in\left(0, x_{0, i}^{*}\right)$. By integration by parts,

$$
\psi_{\lambda_{i}}(x)-\mathrm{e}^{-\gamma(x)} \psi_{\lambda_{i}}^{\prime} \Lambda(x)-\left(\mathrm{e}^{-\gamma(\varepsilon)} \psi_{\lambda_{i}}^{\prime}(\varepsilon) \Lambda(\varepsilon)-\psi_{\lambda_{i}}(\varepsilon)\right)=2 \lambda_{i} \int_{\varepsilon}^{x} \Lambda(s) \psi_{\lambda_{i}}(s) \mathrm{e}^{-\gamma(s)} \mathrm{d} s .
$$

Letting $\varepsilon \rightarrow 0^{+}$, since 0 is an LP endpoint, Lemma 10.4.1 of [8] implies that

$$
\mathrm{e}^{-\gamma}\left[\psi_{\lambda_{i}}^{\prime} \Lambda-\psi_{\lambda_{i}} \Lambda^{\prime}\right](\varepsilon) \rightarrow 0^{+}
$$

By the monotone convergence theorem,

$$
\int_{\varepsilon}^{x} \Lambda(s) \psi_{\lambda_{i}}(s) \mathrm{e}^{-\gamma(s)} \mathrm{d} s \rightarrow \int_{0}^{x} \Lambda(s) \psi_{\lambda_{i}}(s) \mathrm{e}^{-\gamma(s)} \mathrm{d} s
$$

So, we obtain

$$
\psi_{\lambda_{i}}(x)-\mathrm{e}^{-\gamma(x)} \psi_{\lambda_{i}}^{\prime}(x) \Lambda(x)=2 \lambda_{i} \int_{0}^{x} \Lambda(s) \psi_{\lambda_{i}}(s) \mathrm{e}^{-\gamma(s)} \mathrm{d} s
$$

We know that in $\left(0, x_{0, i}\right), \psi_{\lambda_{i}}$ is positive and increasing, so the following inequality holds:

$$
\psi_{\lambda_{i}}(x)-\mathrm{e}^{-\gamma(x)} \psi_{\lambda_{i}}^{\prime}(x) \Lambda(x) \leq 2 \lambda_{i} \psi_{\lambda_{i}}(x) \int_{0}^{x} \Lambda(s) \mathrm{e}^{-\gamma(s)} \mathrm{d} s .
$$

Using the fact that $\psi_{\lambda_{i}}(x)=-\left(1 / 2 \lambda_{i}\right)\left(\mathrm{e}^{-\gamma(x)} \psi_{\lambda_{i}}^{\prime}(x)\right)^{\prime} \mathrm{e}^{\gamma(x)}$ and multiplying by $2 \lambda_{i} \mathrm{e}^{-\gamma(x)}$, we obtain

$$
2 \lambda_{i} \Lambda(x) \mathrm{e}^{-\gamma(x)}\left(\mathrm{e}^{-\gamma(x)} \psi_{\lambda_{i}}^{\prime}(x)\right)+\left(\mathrm{e}^{-\gamma(x)} \psi_{\lambda_{i}}^{\prime}(x)\right)^{\prime}\left(1-2 \lambda_{i} \int_{0}^{x} \Lambda(s) \mathrm{e}^{-\gamma(s)} \mathrm{d} s\right) \geq 0 .
$$

Dividing by $\left(1-2 \lambda_{i} \int_{0}^{x} \Lambda(s) \mathrm{e}^{-\gamma(s)} \mathrm{d} s\right)^{2}$ (which is strictly positive in $\left(0, x_{0, i}\right)$ ), and by noting that $\left(1-2 \lambda_{i} \int_{0}^{x} \Lambda(s) \mathrm{e}^{-\gamma(s)} \mathrm{d} s\right)^{\prime}=-2 \lambda_{i} \Lambda(x) \mathrm{e}^{-\gamma(x)}$, we deduce that

$$
\left(\frac{\mathrm{e}^{-\gamma(x)} \psi_{\lambda_{i}}^{\prime}(x)}{1-2 \lambda_{i} \int_{0}^{x} \Lambda(s) \mathrm{e}^{-\gamma(s)} \mathrm{d} s}\right)^{\prime} \geq 0
$$

Then, for $0<x<y<x_{0, i}$, the following inequality is satisfied:

$$
\mathrm{e}^{-\gamma} \psi_{\lambda_{i}}^{\prime}(0+)<\frac{\mathrm{e}^{-\gamma} \psi_{\lambda_{i}}^{\prime}(x)}{1-2 \lambda_{i} \int_{0}^{x} \Lambda(s) \mathrm{e}^{-\gamma(s)} \mathrm{d} s}<\frac{\mathrm{e}^{-\gamma} \psi_{\lambda_{i}}^{\prime}(y)}{1-2 \lambda_{i} \int_{0}^{y} \Lambda(s) \mathrm{e}^{-\gamma(s)} \mathrm{d} s}<\infty .
$$

The right-hand inequality follows from

$$
\left|\frac{\mathrm{e}^{-\gamma(x)} \psi_{\lambda}^{\prime}(x)}{2 \lambda_{i}}\right|=\left|\int_{x}^{\infty} \mathrm{e}^{-\gamma(y)} \psi_{\lambda_{i}}(y) \mathrm{d} y\right| \leq \int_{x}^{\infty} \mathrm{e}^{-\gamma(y)}\left|\psi_{\lambda_{i}}(y)\right| \mathrm{d} y<\infty \quad \text { for all } x>0 .
$$


It guarantees the integrability of $\psi_{\lambda_{i}}$ because

$$
\begin{aligned}
\int_{0}^{x_{0, i} / 2}\left|\psi_{\lambda_{i}}(s)\right| \mathrm{e}^{-\gamma(s)} \mathrm{d} s & =\int_{0}^{x_{0, i} / 2} \psi_{\lambda_{i}}(s) \mathrm{e}^{-\gamma(s)} \mathrm{d} s \\
& =\frac{\mathrm{e}^{-\gamma} \psi_{\lambda_{i}}^{\prime}(0+)-\mathrm{e}^{-\gamma} \psi_{\lambda_{i}}^{\prime}\left(x_{0, i} / 2\right)}{2 \lambda_{i}} \\
& \leq \mathrm{e}^{-\gamma} \psi_{\lambda_{i}}^{\prime}\left(\frac{x_{0, i}}{2}\right) \frac{2 \lambda_{i} \int_{0}^{x_{0, i} / 2} \Lambda(s) \mathrm{e}^{-\gamma(s)} \mathrm{d} s}{1-2 \lambda_{i} \int_{0}^{x_{0, i} / 2} \Lambda(s) \mathrm{e}^{-\gamma(s)} \mathrm{d} s} \\
& <\infty
\end{aligned}
$$

\section{QSDs and the Yaglom limit}

\subsection{Existence}

In the previous section we showed that $\psi_{\lambda_{0}}$ is a strictly positive $\mu$-integrable function. Then, by standard methods, for instance, as in Theorem 5.2 of [1], the normalized measure defined by $\mathrm{e}^{-\gamma} \psi_{\lambda_{0}}$ is a QSD. From Theorem 2.1, a QSD exists only if it is associated to the value $\lambda^{*}$. Hence, we have proven the following result.

Theorem 4.1. If hypothesis $(H)$ holds then there exists a unique QSD given by

$$
v(\mathrm{~d} x)=\frac{\psi_{\lambda_{0}}(x)}{\left(\mathbf{1}_{(0, \infty)}, \psi_{\lambda_{0}}\right)_{\mu}} \mathrm{d} x .
$$

Moreover, $\lambda_{0}=\lambda^{*}$.

In our case, i.e. one-dimensional diffusions killed at 0 verifying hypothesis $(\mathrm{H})$, we will use the same arguments as in the proof of Theorem 5.3 of [1] to show the existence of a Yaglom limit. To achieve this, we first need to study in detail the behavior of the transition density of the diffusion process. We recall Theorem 2.3 of [1], which states that hypothesis (H1) guarantees that, for all $x>0$ and $t>0$, there exists a density $r(t, x, y)$ that satisfies

$$
\mathrm{E}_{x}\left(f\left(X_{t}\right) \mathbf{1}_{\left\{T_{0}>t\right\}}\right)=\int_{0}^{\infty} r(t, x, y) f(y) \mu(\mathrm{d} y) .
$$

Moreover, we also have the following result on the density $r(t, x, y)$ of (4.1). Under hypothesis $(\mathrm{H})$, the density satisfies

$$
r(t, x, y)=\sum_{k \geq 0} \mathrm{e}^{-\lambda_{k} t} \psi_{\lambda_{k}}(x) \psi_{\lambda_{k}}(y) \quad \text { uniformly on compact sets of }(0, \infty)^{3} .
$$

The proof of this property is similar to that of Proposition 3.3 of [1], since it only uses the discrete spectrum property. Let us state the last required property on the density $r(t, x, y)$, in order to obtain the Yaglom limit property similarly as was done in Theorem 5.2 of [1].

Proposition 4.1. Assume that hypothesis $(H)$ holds. Then $r(t, x, y) \in L^{2}(\mu)$ for all $t>0$ and $x>0$. Moreover, there exists a function $B(t) \geq 0, \lim _{t \rightarrow \infty} B(t)=0$, such that

$$
\int_{0}^{\infty} r^{2}(t, x, y) \mu(\mathrm{d} y)<r(t, x, x) B(t)<\infty \text { for all } t>0, x>0 .
$$


Proof. From relation (4.2) and the Cauchy-Schwartz inequality, we obtain

$$
r^{2}(t, x, y) \leq \sum_{k \geq 0} \mathrm{e}^{-\lambda_{k} t} \psi_{\lambda_{k}}^{2}(x) \sum_{k \geq 0} \mathrm{e}^{-\lambda_{k} t} \psi_{\lambda_{k}}^{2}(y)=r(t, x, x) \sum_{k \geq 0} \mathrm{e}^{-\lambda_{k} t} \psi_{\lambda_{k}}^{2}(y),
$$

where the series are convergent. Moreover, on any compact set $K$ of $\mathbb{R}^{+}$, we obtain

$$
\int_{K} r^{2}(t, x, y) \mu(\mathrm{d} y) \leq r(t, x, x) \int_{K} \sum_{k \geq 0} \mathrm{e}^{-\lambda_{k} t} \psi_{\lambda_{k}}^{2}(y) \mu(\mathrm{d} y) .
$$

By Tonelli's theorem,

$$
\int_{K} \sum_{k \geq 0} \mathrm{e}^{-\lambda_{k} t} \psi_{\lambda_{k}}^{2}(y) \mu(\mathrm{d} y)=\sum_{k \geq 0} \mathrm{e}^{-\lambda_{k} t} \int_{K} \psi_{\lambda_{k}}^{2}(y) \mu(\mathrm{d} y) \leq \sum_{k \geq 0} \mathrm{e}^{-\lambda_{k} t},
$$

since $\left|\psi_{\lambda_{k}}\right|_{L^{2}(\mu)}=1$. On the other hand, we know that $k /\left(2 \int_{0}^{\infty} \mathrm{e}^{-\gamma(s)} \Lambda(s) \mathrm{d} s\right)=k J$ is a lower bound for $\lambda_{k}$, so $\mathrm{e}^{-\lambda_{k} t} \leq \mathrm{e}^{-k J t}$ and, moreover, there exists $k_{0}$ such that $\lambda_{k_{0}} \geq k_{0} J \geq \lambda_{0}$. It follows that

$$
\sum_{k \geq 0} \mathrm{e}^{-\lambda_{k} t} \leq \mathrm{e}^{-\lambda_{0} t}+\cdots+\mathrm{e}^{-\lambda_{k_{0}-1} t}+\sum_{k \geq k_{0}} \mathrm{e}^{-t k J} \leq \mathrm{e}^{-\lambda_{0} t}\left(k_{0}+\frac{1}{1-\mathrm{e}^{-J t}}\right)=B(t) .
$$

We obtain, for any compact set $K$, the inequality

$$
\int_{K} r^{2}(t, x, y) \mu(\mathrm{d} y) \leq r(t, x, x) B(t) .
$$

Since the bound on the right-hand side does not depend on $K$, letting it to tend to $\mathbb{R}^{+}$yields the result.

Theorem 4.2. Assume that hypothesis $(H)$ holds. Then, for all $x>0$ and all Borel subsets $A \subseteq(0, \infty)$,

$$
\begin{gathered}
\lim _{t \rightarrow \infty} \mathrm{e}^{\lambda_{0} t} \mathrm{P}_{x}\left(T_{0}>t\right)=\psi_{\lambda_{0}}(x)\left(\psi_{\lambda_{0}}, 1\right)_{\mu}, \\
\lim _{t \rightarrow \infty} \mathrm{e}^{\lambda_{0} t} \mathrm{P}_{x}\left(X_{t} \in A, T_{0}>t\right)=v(A) \psi_{\lambda_{0}}(x)\left(\psi_{\lambda_{0}}, 1\right)_{\mu} .
\end{gathered}
$$

We also have

$$
\lim _{t \rightarrow \infty} \mathrm{e}^{\lambda_{0} t} \mathrm{P}_{x}\left(X_{t} \in A \mid T_{0}>t\right)=v(A),
$$

that is, $v$ is the Yaglom limit distribution. Moreover, any measure $\rho$ with compact support in $(0, \infty)$ satisfies

$$
\begin{gathered}
\lim _{t \rightarrow \infty} \mathrm{e}^{\lambda_{0} t} \mathrm{P}_{\rho}\left(T_{0}>t\right)=\left(\psi_{\lambda_{0}}, 1\right)_{\mu} \int \psi_{\lambda_{0}} \rho, \\
\lim _{t \rightarrow \infty} \mathrm{e}^{\lambda_{0} t} \mathrm{P}_{x}\left(X_{t} \in A, T_{0}>t\right)=v(A)\left(\psi_{\lambda_{0}}, 1\right)_{\mu} \int \psi_{\lambda_{0}} \rho, \\
\lim _{t \rightarrow \infty} \mathrm{e}^{\lambda_{0} t} \mathrm{P}_{\rho}\left(X_{t} \in A \mid T_{0}>t\right)=v(A) .
\end{gathered}
$$

Proof. The same proof as in [1] works because $r(t, x, y)$ fulfills all the required properties. 


\section{Acknowledgements}

The author acknowledges support from CONICYT PhD fellowship and the MATHAMSUD's project 'Stochastic structure of large interacting systems'. The author is also indebted to the anonymous referees for their valuable comments and corrections that significantly improved this work, and to Servet Martinez and Jaime San Martin for introducing him to the problem studied in this work.

\section{References}

[1] Cattiaux, P. et al. (2009). Quasi-stationary distributions and diffusion models in population dynamics. Ann. Prob. 37, 1926-1969.

[2] Collet, P., Martínez, S. and San Martín, J. (1995). Asymptotic laws for one-dimensional diffusions conditioned to nonabsorption. Ann. Prob. 23, 1300-1314.

[3] Ferrari, P. A., Martínez, S. and Picco, P. (1992). Existence of nontrivial quasi-stationary distributions in the birth-death chain. Adv. Appl. Prob. 24, 795-813.

[4] Funushima, M. (1980). Dirichlet Forms and Markov Processes (North-Holland Math. Library 23). NorthHolland, Amsterdam.

[5] IKeda, N. And Watanabe, S. (1989). Stochastic Differential Equations and Diffusion Processes (North-Holland Math. Library 24), 2nd edn. North-Holland, Amsterdam.

[6] Karlin, S. and Taylor, H. M. (1981). A Second Course in Stochastic Processes, 2nd edn. Academic Press, New York.

[7] Kolb, M. and Steinsaltz, D. (2012). Quasi limiting behavior for one dimensional diffusions with killing. To appear in Ann. Prob.

[8] Zettl, A. (2005). Sturm-Loiuville Theory (Math. Surveys Monogr. 121). American Mathematical Society, Providence, RI. 